PAPER

\title{
Altered cerebellar functional connectivity mediates potential adaptive plasticity in patients with multiple sclerosis
}

\author{
S Saini, N DeStefano, S Smith, L Guidi, M P Amato, A Federico, P M Matthews
}

J Neurol Neurosurg Psychiatry 2004;75:840-846. doi: 10.1136/jnnp.2003.016782

See end of article for authors' affiliations ....................

Correspondence to: Professor P M Matthews, Centre for Functional Magnetic Resonance Imaging of the Brain, John Radcliffe Hospital, Headley Way, Headington, Oxford OX3 9DU, UK; paul@fmrib.ox.ac.uk

Received 13 April 2003 In revised form 4 August 2003 Accepted

10 September 2003

\begin{abstract}
Background: The cerebellum is of potential interest for understanding adaptive responses in motor control in patients with multiple sclerosis because of the high intrinsic synaptic plasticity of this brain region.

Objective: To assess the relative roles of interactions between the neocortex and the cerebellum using measures of functional connectivity.

Methods: A role for altered neocortical-cerebellar functional connectivity in adaptive responses to injury from multiple sclerosis was tested using 1.5 T functional magnetic resonance imaging (fMRI) during figure writing with the dominant right hand in patients with predominantly early relapsing-remitting multiple sclerosis.

Results: Patients $(n=14)$ showed a more bihemispheric pattern of activation in motor cortex than healthy controls $(n=11)$. Correlations between task related signal changes in neocortical and cerebellar regions of interest were used as a measure of functional connectivity. Healthy controls showed strong functional connectivity between the left motor cortex and the right cerebellar dentate nucleus. Significant connectivity between the left primary motor cortex and the right dentate was not found in patients. However, patients had significant connectivity between the left premotor neocortex and the ipsilateral (left) cerebellar cortex (crus I), which was not found in healthy controls.

Conclusions: Changes in apparent cerebellar-neocortical functional connectivity may mediate potentially adaptive changes in brain motor control in patients with multiple sclerosis. Similar changes in the cerebellum and premotor cortex have been reported in the healthy brain during motor learning, suggesting that common mechanisms may contribute to normal motor learning and motor recovery affer injury from multiple sclerosis.
\end{abstract}

this by assessing correlations between signal intensity changes in selected neocortical and cerebellar regions of interest.

\section{METHODS \\ Clinical details}

We recruited 11 right handed healthy controls (median age 37 years (range 27 to 43 ); five male, six female) and 14 right handed outpatients with clinically stable relapsing-remitting multiple sclerosis (median age 37 years (range 18 to 52 ); five male, nine female; mean disease duration 3.6 years (range 1 to 8$)$ ). Patient disability was minimal as assessed by the expanded disability status score (EDSS) (median EDSS, 1.0 (range 1.0 to 2.5$)$ ). Their clinical features are given in table 1 . All patients had been relapse-free for at least three months before the study. No patients had tremor or ataxia. None had weakness or sensory symptoms, or signs affecting the right hand.

All subjects gave their informed consent for participation in the study, which was approved by the ethics committee of the faculty of medicine in the University of Siena, Italy.

\section{Functional assessment}

Patients showed no symptoms or clinical signs of upper limb impairment and were able to perform simple figure writing as well as the controls. Before entering the scanner, patients and controls traced around five predrawn figure $8 \mathrm{~s}$ as accurately

Abbreviations: EDSS, expanded disability status score; FILM, FMRIB's improved linear model; FLAME, FMRIB's local analysis of mixed effects; FMRIB, Oxford Centre for Functional Magnetic Resonance Imaging of the Brain; LI, lateralisation index; MCFLIRT, motion correction using FMIRB's linear image registration tool; ROI, region of interest connectivity with a skilled but overlearned hand movement (figure writing) in patients with multiple sclerosis. We did 
Table 1 Clinical characteristics of patients with multiple sclerosis

\begin{tabular}{|c|c|c|c|c|c|c|c|}
\hline Patient & Sex & $\begin{array}{l}\text { Age } \\
\text { (years) }\end{array}$ & $\begin{array}{l}\text { Disease duration } \\
\text { (years) }\end{array}$ & EDSS & $\begin{array}{l}\text { Lesion volume } \\
\left(\mathrm{cm}^{3}\right)\end{array}$ & $\begin{array}{l}\text { Brain volume } \\
\left(/ 10^{4}\right)\left(\mathrm{cm}^{3}\right)\end{array}$ & Performance product \\
\hline 1 & $M$ & 29 & 6 & 1.5 & 7.55 & 1.69 & 168.2 \\
\hline 2 & $M$ & 18 & 0.6 & 1 & 1.19 & 1.60 & 219.5 \\
\hline 3 & $\mathrm{~F}$ & 37 & 0.1 & 1.5 & 0.23 & 1.61 & 109.3 \\
\hline 4 & $\mathrm{~F}$ & 45 & 6 & 2.5 & 15.40 & 1.50 & 1186.1 \\
\hline 5 & $\mathrm{~F}$ & 40 & 4 & 1 & 1.43 & 1.57 & 126.8 \\
\hline 6 & $\mathrm{~F}$ & 41 & 6 & 1 & 11.03 & 1.58 & 138.5 \\
\hline 7 & $\mathrm{~F}$ & 39 & 3.5 & 1 & 3.01 & 1.50 & 106.6 \\
\hline 8 & $\mathrm{~F}$ & 30 & 2 & 1 & 12.40 & 1.60 & 132.1 \\
\hline 9 & $\mathrm{~F}$ & 37 & 2.5 & 1.5 & 11.03 & 1.59 & 182.7 \\
\hline 10 & $M$ & 27 & 1 & 1.5 & 0.92 & 1.72 & 26.4 \\
\hline 11 & $M$ & 30 & 1 & 1 & 2.18 & 1.66 & 65.5 \\
\hline 12 & $\mathrm{~F}$ & 34 & 8 & 1 & 0.51 & 1.64 & 42.6 \\
\hline 13 & $\mathrm{~F}$ & 52 & 3.3 & 2.5 & 0.32 & 1.59 & 11.9 \\
\hline 14 & $M$ & 45 & 6 & 2.5 & 13.80 & 1.41 & 78.4 \\
\hline Mean (SD) & & $36(9)$ & $3.6(3.4)$ & $1.5(0.6)$ & $5.7(5.7)$ & $1.59(0.10)$ & 185.32 (294.17) \\
\hline Control mean (SD) & & $35(5)$ & & & & $1.61(0.04)$ & $3.6(1.5)$ \\
\hline
\end{tabular}

Performance product: the mean area covered outside of the predrawn figures and the mean time taken to draw around them were measured to provide an index of subject performance (performance product) $=$ mean time $\times$ mean area.

EDSS, extended disability status score; $F$, female; $M$, male.

and as quickly as possible. The mean area covered outside of the predrawn figures and the mean time taken to draw around them were measured to provide an index of subject performance (performance product $=$ mean time $\times$ mean area).

\section{fMRI scanning and paradigm}

Imaging was undertaken with a Philips Gyroscan ACS NT $1.5 \mathrm{~T}$ scanner. BOLD multishot echo-planar images were acquired (time of repetition $(\mathrm{TR})=3000 \mathrm{~ms}$, time of echo $(\mathrm{TE})=40 \mathrm{~ms}$, flip angle $=90^{\circ}, 20$ contiguous axial slices of 7 $\mathrm{mm}$ thickness acquired parallel to a line defined by the anterior and posterior commissures and including brain from the base of the cerebellum to the vertex, field of view (FOV) $250 \times 250 \mathrm{~mm}^{2}, 128 \times 128$ matrix). High resolution whole brain structural scans were acquired using a $\mathrm{Tl}$ weighted sequence $\left(\mathrm{TR}=20 \mathrm{~ms}, \mathrm{TE}=3 \mathrm{~ms}\right.$, flip angle $=20^{\circ}, 25$ contiguous slices of $5 \mathrm{~mm}$ thickness acquired parallel to the AC-PC plane). Turbo spin echo T2 weighted scans were also obtained for each subject $(\mathrm{TR}=2075 \mathrm{~ms}, \mathrm{TE}=90 \mathrm{~ms}$, FOV $=250 \times 250 \mathrm{~mm}^{2}, 256 \times 256$ matrix, signal average $=1$, providing 50 contiguous slices of $3 \mathrm{~mm}$ thickness acquired parallel to the AC-PC plane).

Subjects lying supine in the scanner were asked to write " 8 " repeatedly on paper in a cursive manner (without lifting the hand) for 30 seconds with a pencil in their right hand, to complete a figure every second. This was alternated with 30 second periods of rest. During both conditions the subjects were asked to fix their gaze on a red flashing light cue positioned at the end of the magnet. Each subject undertook six paired rest and writing blocks for a single functional scan and had six functional scans during each session. All subjects were monitored visually throughout the scanning for nontask-related movements.

\section{Image analysis}

The data were processed and analysed using advanced image analysis tools from the Oxford Centre for Functional Magnetic Resonance Imaging of the Brain (FMRIB) Software Library (available at http://www.fmrib.ox.ac.uk/ fsl). Before statistical analysis, motion correction using MCFLIRT, ${ }^{17}$ spatial smoothing (Gaussian kernal of $5 \mathrm{~mm}$ full width at half maximum), intensity normalisation, and non-linear high pass temporal filtering were applied. Statistical analysis was done using the general linear model with FMRIB's improved linear model (FILM) ${ }^{18}$ to generate activation maps expressing relative signal change in active versus rest blocks. Cluster detection was undertaken on voxels at a threshold of $z>2.3$. A probability threshold of $\mathrm{p}<0.01$ was then applied to determine significant clusters.

Using FLIRT, ${ }^{17}$ the functional images were registered to the high resolution $\mathrm{Tl}$ weighted scan and transformed into a standard brain space based on the MNI 152 template. ${ }^{19}$ Mixed effects analysis using FLAME ${ }^{20}$ was used to contrast activation patterns between groups.

\section{Generation of ROI masks}

Regions of interest (ROI) were defined bilaterally on each individual's high resolution Tl weighted structural image using anatomical landmarks identified on the Tl weighted structural scan (fig 1):

- Primary motor cortex (Ml), defined as the posterior half of the precentral gyrus, extending on the lateral surface of the cortex to the midline and from the vertex of the brain to the level of the superior aspect of the lateral ventricles caudally.

- Premotor cortex (PM), defined as the anterior half of the precentral gyrus, the precentral sulcus, and the middle frontal gyrus up to the lateral fissure anteriorly, the lateral surface of the cortex to the midline and from the vertex of the brain to the level of the superior aspect of the lateral ventricles caudally.

- Crus I, defined in the lateral cerebellar hemisphere, ${ }^{21}$ extending rostro-caudally between the superior and inferior aspects of the dentate nucleus.

- Dentate (DN), defined as midline and paravermian central to cerebellar white matter. ${ }^{21}$

\section{Calculation of lesion volume}

Lesion volumes were semimanually defined on T2 weighted axial scans for each patient using edge thresholding. ${ }^{22}$ Hyperintense lesion volumes of more than $3 \mathrm{~mm}$ in diameter were summed to provide a total brain lesion volume (table 1).

\section{Calculations of brain volume}

Measurements of brain size (normalised for head size) were made for each patient on Tl weighted structural scans as an estimate of disease burden related atrophy, using SIENAX, a fully automated tool ${ }^{23}$ (table 1). 

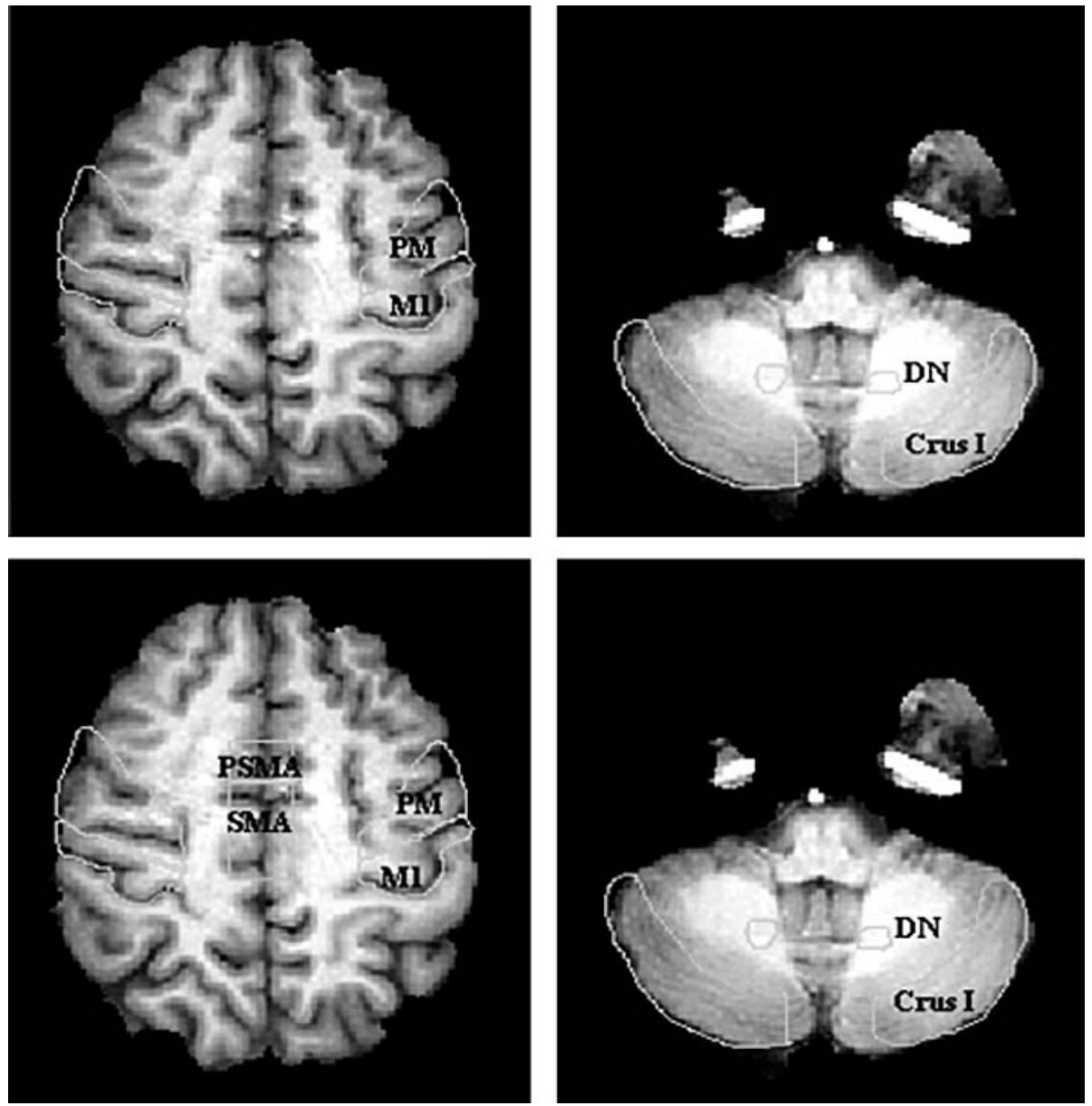

Figure 1 Representative regions of interest (ROI) drawn on axial slices of one subjects $\mathrm{Tl}$ structural scan. The ROI were outlined on several slices for each subject to include areas of interest in a rostral-caudal direction. Crus I, lateral cerebellar hemisphere; DN, dentate nucleus region; MI, primary motor cortex; PM, premotor cortex; PSMA presupplementary motor area; SMA, supplementary motor area.

\section{Data analysis}

The mean positive signal intensity change in each ROI was obtained from the parameter estimates image for each subject. This allowed the calculation of a lateralisation index $(\mathrm{LI}=[\mathrm{C}-\mathrm{I}] /[\mathrm{C}+\mathrm{I}]$, where $\mathrm{C}=$ mean contralateral and $\mathrm{I}=$ mean ipsilateral signal intensity change) for Ml, PM, crus I, and DN regions. The LI has values between 1 (positive signal change only in the side contralateral to the hand moved) and -1 (positive signal change only in the side ipsilateral to the hand moved). To assess whether activation was significantly less lateralised in patients than in control subjects, two tailed Mann-Whitney tests were used to compare signal intensity changes and LIs for each ROI. Relations between cortical and cerebellar signal intensity change were assessed for correlations using a Pearson correlation coefficient. As described in previous reports (see, for example, Koski and Paus $^{24}$ and Horwitz et a ${ }^{25}$ ), such correlations can be used as measures of functional connectivity between brain regions. A correction for multiple comparisons was applied.

\section{RESULTS}

We studied patients with predominantly early relapsingremitting multiple sclerosis, along with age matched, healthy controls. The lesion load was modest (mean $5.7 \mathrm{~cm}^{3}$; range 0.2 to $\left.15.4 \mathrm{~cm}^{3}\right)$ for the patients $(\mathrm{n}=14)$, and their mean normalised brain volume was similar to that of the controls (mean (SD) brain volume of patients, $1.59 \times 10^{3}(100) \mathrm{cm}^{3}$; controls, $\left.1.61 \times 10^{3}(40) \mathrm{cm}^{3}\right)$. Task performance was assessed by a timed figure writing trial. There was no difference in either time $(t=-0.774, \mathrm{df}=23, \mathrm{p}=0.447)$, area included between the template and traced lines $(t=0.054, \mathrm{df}=23$, $\mathrm{p}=0.958)$, or performance product $(t=-0.71 \mathrm{l}, \mathrm{df}=23$, $\mathrm{p}=0.484$ ) between controls and patients. No "mirror" movements of the contralateral hand were observed during the writing task for any of the patients.

Both patients and controls activated a distributed network of regions associated with movement, including the motor cortex and cerebellum (fig 2; table 2). There were no differences in localisation between centres of activation in patients and controls. However, consistent with previous studies, ${ }^{7}$ patients showed a more bihemispheric pattern of motor cortex activation. This hemispheric activation asymmetry was assessed quantitatively for premotor (PM) and primary motor $(\mathrm{Ml})$ cortical regions of interest. There was a reduction of over $50 \%$ in the PM LI in patients $(\mathrm{U}=32.50$, $\mathrm{p}=0.013$ ) (mean (SD) PM LI for controls, 0.37 (0.18); mean PM LI for patients, 0.18 (0.18); (fig 3)). This difference in LI primarily was caused by increased signal intensity changes in the right premotor cortex in patients relative to controls $(\mathrm{U}=29, \mathrm{p}=0.008)$. Ml activation lateralisation was not significantly different between patients and controls (mean M1 LI for controls, 0.60 (0.10); mean Ml LI for patients, 0.54 $(0.13))$.

The relations between the relative changes in cerebral cortex and cerebellar signal intensities within individual 
subjects offer measures of neocortical-cerebellar functional connectivity (see, for example, Koski and Paus ${ }^{24}$ and Horwitz et $\left.a l^{25}\right)$. These were different between patients and controls (table 3). The control group showed the expected $^{26} 27$ strong "crossed" correlation between activation changes in the left $\mathrm{Ml}$ and right dentate ROI, which was not found in multiple sclerosis patients. No ipsilateral correlations were found in controls, but patients showed a significant ipsilateral correlation between the left PM cortex and the left cerebellar hemisphere (crus I) (table 3).

No significant relation was found between either LI or regional signal changes and brain volumes, lesion volumes, or EDSS.

\section{DISCUSSION}

This study extends the description of changes that occur in brain motor control in response to injury from multiple sclerosis. Along with other recent reports, ${ }^{8}{ }^{10} 28$ it emphasises that functional changes occur early in the clinical course of multiple sclerosis, and that they need not be accompanied by identifiable performance deficits. As found in similar previous studies, the patients with multiple sclerosis showed increased ipsilateral premotor activation relative to controls. ${ }^{7828}$ What is novel here is the characterisation of the associated, altered neocortical-cerebellar functional connectivity. Increased connectivity was found in patients with multiple sclerosis between the premotor cortex contralateral to the hand moved and the cerebellar cortex (the input region for the cortico-ponto-cerebellar projection system) on the same side, with decreased connectivity relative to healthy controls for the crossed cerebellar output (dentate) projections to the same premotor cortex..$^{29}$ Potentially adaptive responses to injury thus affect not only activity in specific regions, but also interactions between regions in the distributed network for motor control. The results suggest a role for the cerebellum in mediating adaptive changes to injury from multiple sclerosis.
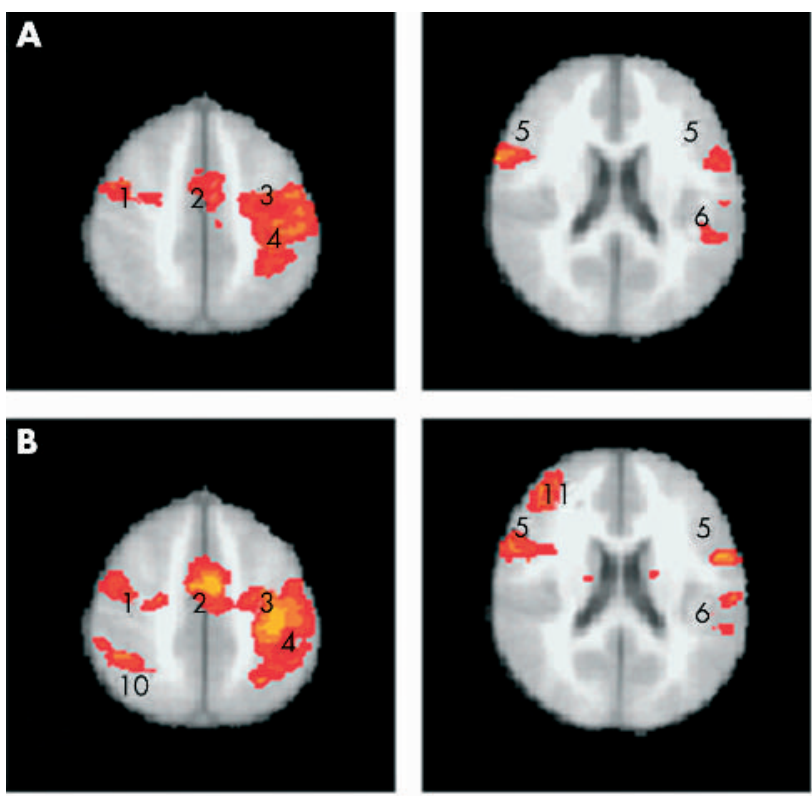

$Z=48$

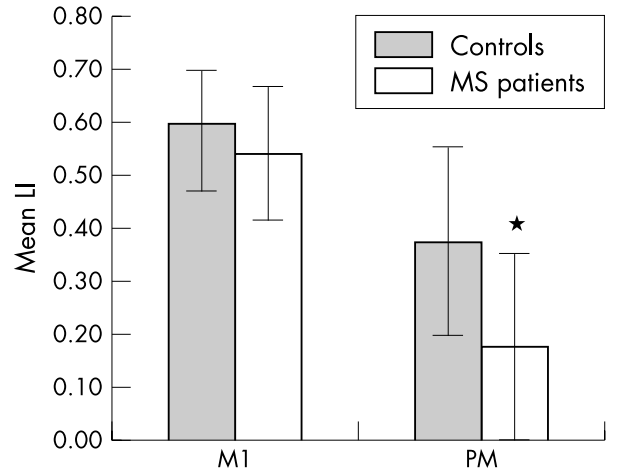

Figure 3 Comparison of mean lateralisation indices (LI) between control and patients groups. Patients had a significantly lower LI (more bilateral activation) in PM compared with the control group ( ${ }^{*} p=0.013$ ). M1, primary motor cortex; PM, premotor cortex. Error bars show standard deviations.

The cerebellum may have a special role in forms of brain functional plasticity. ${ }^{30-32}$ Cerebellar connections allow rapid, activity dependent alterations in synaptic efficiency. ${ }^{33} 34$ Functional imaging has defined increases in cerebellar activation with learning that involve both the cerebellar hemispheres and the dentate nucleus, the origin of efferent dentato-thalamo-cortical and dentato-thalamo-striate tracts. ${ }^{29}$ A notable aspect of earlier stages of motor learning is increased recruitment of the cerebellum bilaterally, in a similar fashion to the pattern found here for patients. ${ }^{14}$ Damage to the cerebellum impairs forms of implicit learning. ${ }^{33}{ }^{35}$ Increased cerebellar activity is associated with better outcome in patients after stroke. ${ }^{36}$

An increase in functional connectivity to the cerebellar hemisphere contralateral to the hand moved was observed here with the patients. Increases in activity in the contralateral cerebellar hemisphere are also found with motor learning in healthy subjects. ${ }^{14}$ This may reflect recruitment
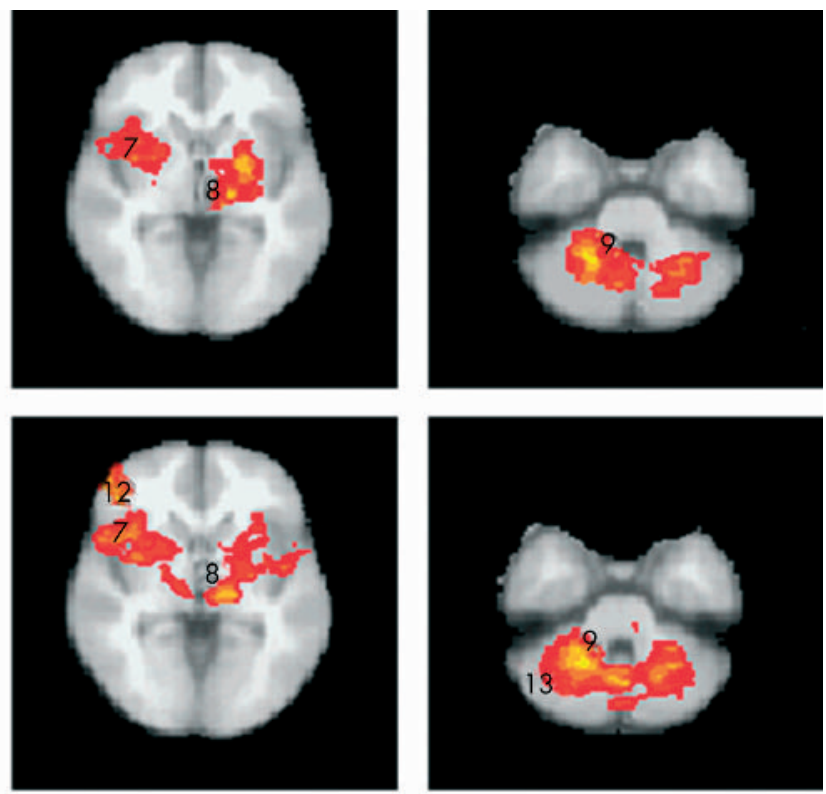

$Z=-20$

$Z=-32$

Figure 2 Mean random effects statistical activation maps for (A) control and (B) multiple sclerosis patient groups, demonstrating regions of significant activation during writing $(z>2.3, p=0.01$, corrected). Activation is averaged over six runs for each subject and superimposed on a standard space image. Numbers represent: 1, middle frontal gyrus; 2 , medial frontal gyrus (supplementary motor area); 3 , precentral gyrus; 4 , postcentral gyrus; 5 , inferior frontal gyrus; 6, inferior parietal lobule; 7 , putamen; 8 , thalamus; 9 , dentate nucleus; 10, superior parietal lobule; 11, superior frontal gyrus; 12 , inferior frontal gyrus; 13 , crus I. L, left, R, right. 
Table 2 Anatomical coordination in standard brain space and maximum $z$ scores for activated voxels in controls $(n=11)$ and patients $(n=14)$ during writing from mixed effect group analyses

\begin{tabular}{|c|c|c|c|c|c|c|c|c|}
\hline \multirow[b]{3}{*}{ Anatomical regions } & \multicolumn{8}{|c|}{ Coordinates of voxels with maximum z score } \\
\hline & \multicolumn{4}{|c|}{ Controls } & \multicolumn{4}{|c|}{ Patients } \\
\hline & $\mathbf{x}$ & $y$ & $\mathbf{z}$ & $\operatorname{Max} z$ & $\mathbf{x}$ & $\mathbf{Y}$ & $\mathbf{z}$ & Max z \\
\hline \multicolumn{9}{|l|}{ Cortical } \\
\hline L precentral gyrus & -32 & -14 & 52 & 6.00 & -38 & -20 & 54 & 10.93 \\
\hline $\mathrm{R}$ precentral gyrus & 54 & 2 & 44 & 5.84 & 40 & -2 & 60 & 6.97 \\
\hline L postcentral gyrus & -36 & -30 & 66 & 8.28 & -54 & -20 & 38 & 10.88 \\
\hline Pre-SMA & & & & & 8 & 2 & 56 & 8.95 \\
\hline SMA proper & -6 & -2 & 52 & 5.84 & -10 & -6 & 58 & 9.04 \\
\hline $\mathrm{R}$ middle frontal gyrus (posterior) & -54 & 2 & 40 & 8.32 & 54 & 8 & 34 & 8.94 \\
\hline $\mathrm{R}$ middle frontal gyrus (anterior) & & & & & 44 & 36 & 24 & 7.05 \\
\hline $\mathrm{L}$ inferior frontal gyrus & & & & & -56 & 6 & 26 & 9.01 \\
\hline $\mathrm{R}$ inferior frontal gyrus & 64 & 10 & 20 & 8.08 & 54 & 6 & 26 & 7.06 \\
\hline $\mathrm{R}$ lateral orbital gyrus & & & & & 48 & 48 & -6 & 8.90 \\
\hline L supramarginal gyrus & -46 & -38 & 54 & 5.76 & -46 & -32 & 36 & 5.12 \\
\hline$L$ insula & & & & & -36 & 0 & -10 & 5.10 \\
\hline $\mathrm{R}$ insula & 42 & 14 & -6 & 5.71 & 36 & 4 & -6 & 5.09 \\
\hline L angular gyrus & & & & & -32 & -56 & 52 & 5.03 \\
\hline \multicolumn{9}{|l|}{ Subcortical } \\
\hline $\mathrm{L}$ thalamus & -18 & -18 & 0 & 5.96 & -20 & -20 & 0 & 7.11 \\
\hline $\mathrm{R}$ thalamus & & & & & 10 & -16 & -2 & 5.09 \\
\hline $\mathrm{L}$ thalamus & & & & & -14 & -2 & 10 & 5.19 \\
\hline L globus pallidus & -20 & -2 & 8 & 6.04 & -24 & -6 & 0 & 5.18 \\
\hline R globus pallidus & 32 & 0 & 4 & 5.94 & 20 & -2 & 8 & 5.21 \\
\hline L putamen & -22 & 0 & -2 & 8.47 & -18 & 14 & -14 & 5.13 \\
\hline $\mathrm{R}$ putamen & 24 & 2 & 2 & 5.94 & 32 & 4 & 0 & 5.11 \\
\hline L cerebellar hemisphere (crus I) & -26 & -68 & -32 & 5.87 & -34 & -62 & -38 & 5.11 \\
\hline R cerebellar hemisphere (crus I) & 18 & -70 & -30 & 8.35 & 18 & -66 & -30 & 3.01 \\
\hline $\mathrm{L}$ dentate nucleus & -30 & -50 & -40 & 6.02 & -28 & -54 & -38 & 7.12 \\
\hline $\mathrm{R}$ dentate nucleus & 24 & -48 & -32 & 13.47 & 20 & -52 & -36 & 11.00 \\
\hline Vermis & 6 & -54 & -16 & 8.29 & 6 & -50 & -22 & 8.95 \\
\hline
\end{tabular}

of uncrossed ponto-cerebellar projections. In studies of the cat, while the majority of ponto-cerebellar projections are crossed, almost a quarter were found to project to the same side. ${ }^{37}$ This provides a pathway for input from the motor cortex to the cerebellar hemisphere on the same side.

The motor cortex, cerebellum, thalamus, substantia nigra, and basal ganglia together constitute key elements of a distributed motor control network. Subcortical structures project widely to neocortical regions involved in motor control. ${ }^{38}$ The increased functional connectivity between the motor cortex and cerebellum found in the patients involved the premotor and not the primary motor cortex. Changes in relative premotor cortex activity in the patients account for the more bihemispheric pattern of motor cortex activation in the patients. Previous studies of patients after stroke or neurorehabilitation have suggested the particular importance of activity changes in the premotor cortex to functional recovery. ${ }^{11} 39$

Observation on the changes in functional connectivity and patterns of premotor activity may be related. Cerebellar activation contralateral to the hand moved provides a potential pathway by which premotor cortex ipsilateral to the hand moved might be recruited for motor control, through crossed dentate-cortical projections. A subcortical pathway linking activity in motor cortices of the two hemispheres is suggested by observations such as those showing that motor cortex activation ipsilateral to the hand moved does not require an intact corpus callosum. ${ }^{40}{ }^{41}$ However, the directionality of the functional interactions between cerebellum and premotor cortex cannot be defined uniquely on the basis of the functional connectivity data

Table 3 Correlations (showing $r$ values and significance) between signal intensity changes in specified regions of interest in the motor cortex and cerebellum for healthy controls and patients

\begin{tabular}{|c|c|c|c|c|}
\hline \multirow[b]{2}{*}{ Correlation } & \multicolumn{2}{|c|}{ Controls } & \multicolumn{2}{|c|}{ Patients } \\
\hline & $r$ & $\mathrm{p}$ Value & $r$ & p Value \\
\hline \multicolumn{5}{|c|}{ Crossed pathways } \\
\hline L MI-R DN & 0.79 & 0.024 & 0.42 & 0.840 \\
\hline LPM-R DN & 0.59 & 0.360 & 0.53 & 0.300 \\
\hline L PM-R crus I & 0.29 & 0.380 & 0.62 & 0.120 \\
\hline \multicolumn{5}{|c|}{ Uncrossed pathways } \\
\hline R PM-R crus I & -0.02 & 0.946 & 0.59 & 0.280 \\
\hline LPM-L crus I & 0.07 & 0.840 & 0.73 & 0.024 \\
\hline R Ml-R crus I & 0.03 & 0.940 & 0.66 & 0.060 \\
\hline
\end{tabular}


here. There are alternative models consistent with the data. One possibility, for example, is a direct primary interaction between the motor cortices of the two hemispheres, which in turn drives more bilateral cerebellar activity-although this may provide a less compelling explanation, given that the patients also show decreased functional connectivity between the cerebellar activation ipsilateral to, and neocortical activity contralateral to, the hand moved.

There are a few technical issues that should be considered in the interpretation of these data. First, the use of such a relatively mildly affected and homogeneous group (as well as its small size) limits confounding from differences in performance, but precludes adequate testing of the relations between activation in specific brain areas and clinical variables such as disease burden, duration, and disability. Not unexpectedly, correlations between functional changes and these measures were not found (data not shown). Second, early reports argued that increased ipsilateral motor cortex activity in patients may reflect "mirror" movements, raising this as a concern here. ${ }^{42}$ Our patients (like other patient groups ${ }^{73}$ ) showed no clinical evidence of mirror movements. Third, in previous studies, we used the relative number of significantly activated voxels for calculation of $\mathrm{LI}^{7}{ }^{7}$ However, as the absolute number of activated voxels is a function of the statistical threshold chosen, absolute values of such LIs are not interpreted easily and may have higher variance because of dependence on noise in the near threshold voxels. ${ }^{44}$ These problems were limited by calculation of LI from relative signal intensity changes. $^{45}$

Involvement of multiple processing regions of a distributed motor network in adaptive compensation for the brain injury of multiple sclerosis implies that optimising recovery demands optimising functions of multiple targets and their interactions. Altering levels of diffusely projecting modulatory neurotransmitters that may affect interactions between brain regions is thus a theoretically attractive approach. ${ }^{46}$ Second, because of the distributed nature of control, the relation between lesion distribution and functional impairment may be expected to be complex. In fact, previous work has shown only minimal gains in the strengths of correlations between measures of functional impairment in the motor system and lesion burden when the volume in the corticospinal tract alone is used rather than global brain lesion load. ${ }^{47}$ Together, these observations suggest that in established disease the focus for repair of injury should be on the global rather than the system specific disease burden. Finally, the recognition that interactions between spatially distant nodes in a coordinated network may promote recovery emphasises that in multiple sclerosis, a disease characterised by substantial diffuse axonal destruction, the potential mechanisms of recovery are impaired with progression of the disease, just as are primary effector pathways.

\section{ACKNOWLEDGEMENTS}

PMM gratefully acknowledges support from the MS Society of Great Britain and Northern Ireland and the Medical Research Council (UK). NDS was supported by a PAR grant to the University of Siena. This work was conducted as part of the Brain Imaging Laboratory Linkage involving the Universities of Oxford and Siena.

\footnotetext{
Authors' affiliations

S Saini, S Smith, P M Matthews, Centre for Functional Magnetic Resonance Imaging of the Brain, John Radcliffe Hospital, University of Oxford, Oxford, UK

N DeStefano, A Federico, Department of Neurology and Behavioural Sciences, University of Siena, Siena, Italy

L Guidi, Unit of Neurology, Empoli Hospital, Empoli, Italy
}

M P Amato, Department of Neurology, University of Florence, Florence, Italy

Competing interests: none declared

\section{REFERENCES}

1 Medana IM, Esiri MM. Axonal damage: a key predictor of outcome in human CNS diseases. Brain 2003; 126:515-30.

2 Matthews PM, De Stefano N, Narayanan S, et al. Putting magnetic resonance spectroscopy studies in context: axonal damage and disability in multiple sclerosis. Semin Neurol 1998;18:327-36.

3 Bjartmar C, Wujek JR, Trapp BD. Axonal loss in the pathology of MS: consequences for understanding the progressive phase of the disease. J Neurol Sci 2003;206: 165-71.

4 Dijkhuizen RM, Singhal AB, Mandeville JB, et al. Correlation between brain reorganization, ischemic damage, and neurologic status after transient focal cerebral ischemia in rats: a functional magnetic resonance imaging study. J Neurosci 2003;23:510-17.

5 Cramer SC, Nelles G, Benson RR, et al. A functional MRI study of subjects recovered from hemiparetic stroke. Stroke 1997;28:2518-27.

6 Marshall RS, Perera GM, Lazar RM, et al. Evolution of cortical activation during recovery from corticospinal tract infarction. Stroke 2000;31:656-61

7 Lee $\mathrm{M}$, Reddy $\mathrm{H}$, Johansen-Berg $\mathrm{H}$, et al. The motor cortex shows adaptive functional changes to brain injury from multiple sclerosis. Ann Neurol 2000;47:606-13.

8 Reddy H, Narayanan S, Matthews PM, et al. Relating axonal injury to functional recovery in MS. Neurology 2000;54:236-9.

9 Pantano P, lannetti GD, Caramia F, et al. Cortical motor reorganization after a single clinical attack of multiple sclerosis. Brain 2002; 125:1607-15.

10 Rocca MA, Falini A, Colombo B, et al. Adaptive functional changes in the cerebral cortex of patients with nondisabling multiple sclerosis correlate with the extent of brain structural damage. Ann Neurol 2002;51:330-9.

11 Johansen-Berg H, Rushworth MF, Bogdanovic MD, et al. The role of ipsilateral premotor cortex in hand movement after stroke. Proc Natl Acad Sci USA 2002;99:14518-23.

12 Rocca MA, Matthews PM, Caputo D, et al. Evidence for widespread movement-associated functional MRI changes in patients with PPMS Neurology 2002; 58:866-72.

13 Voogd J, Glickstein M. The anatomy of the cerebellum. Trends Neurosci 1998;21:370-5.

14 Doyon J, Song AW, Karni A, et al. Experience-dependent changes in cerebellar contributions to motor sequence learning. Proc Natl Acad Sci USA 2002;99:1017-22.

15 Lafleur MF, Jackson PL, Malouin F, et al. Motor learning produces parallel dynamic functional changes during the execution and imagination of sequential foot movements. Neuroimage 2002;16:142-57.

16 Ungerleider LG, Doyon J, Karni A. Imaging brain plasticity during motor skill learning. Neurobiol Learn Mem 2002;78:553-64.

17 Jenkinson M, Smith S. A global optimisation method for robust affine registration of brain images. Med Image Anal 2001;5:143-56.

18 Woolrich MW, Ripley BD, Brady M, et al. Temporal autocorrelation in univariate linear modeling of FMRI data. Neuroimage $2001 ; 14: 1370-86$

19 Evans A, Collins D, Holmes C. Computational approaches to quantifying human neuroanatomical variability. In: Mazziotta J, Toga A, eds. Brain mapping: the methods. New York: Academic Press, 1996:343-61.

20 Behrens T, Woolrich MW, Smith S. Multi-subject null hypothesis testing using a fully Bayesian framework: theory. Human Brain Mapping [in press].

21 Schmahmann JD, Doyon J, Toga A, et al. MRI atlas of the human cerebellum. Syngress Media, 2000.

22 Narayanan S, Fu L, Pioro E, et al. Imaging of axonal damage in multiple sclerosis: spatial distribution of magnetic resonance imaging lesions. Ann Neurol 1997;41:385-91

23 Smith SM, Zhang $Y$, Jenkinson $M$, et al. Accurate, robust, and automated longitudinal and cross-sectional brain change analysis. Neuroimage 2002;17:479-89.

24 Koski L, Paus T. Functional connectivity of the anterior cingulate cortex within the human frontal lobe: a brain-mapping meta-analysis. Exp Brain Res 2000; 133:55-65.

25 Horwitz B, Rumsey JM, Donohue BC. Functional connectivity of the angular gyrus in normal reading and dyslexia. Proc Natl Acad Sci USA 1998;95:8939-44.

26 DiPiero V, Chollet F, Dolan RJ, et al. The functional nature of cerebellar diaschisis. Stroke 1990;21:1365-9.

27 Meyer BU, Roricht S, Machetanz J. Reduction of corticospinal excitability by magnetic stimulation over the cerebellum in patients with large defects of one cerebellar hemisphere. Electroencephalogr Clin Neurophysiol 1994;93:372-9.

28 Pantano P, Mainero C, lannetti GD, et al. Contribution of corticospinal tract damage to cortical motor reorganization after a single clinical attack of multiple sclerosis. Neuroimage 2002;17:1837-43.

29 Brodal P, Bjaalie JG. Salient anatomic features of the cortico-ponto-cerebellar pathway. Prog Brain Res 1997; 1 14:227-49.

30 Schmahmann JD. An emerging concept. The cerebellar contribution to higher function. Arch Neurol 1991;48:1178-87. 
31 Kleim JA, Freeman JH, Bruneau R, et al. Synapse formation is associated with memory storage in the cerebellum. Proc Natl Acad Sci USA 2002;99:13228-31

32 Anderson BJ, Alcantara AA, Greenough WT. Motor-skill learning: changes in synaptic organization of the rat cerebellar cortex. Neurobiol Learn Mem 1996:66:221-9.

$33 \mathrm{Kim}$ JJ, Thompson RF. Cerebellar circuits and synaptic mechanisms involved in classical eyeblink conditioning. Trends Neurosci 1997;20:177-81.

34 Ito $M$. Synaptic plasticity in the cerebellar cortex and its role in motor learning. Can J Neurol Sci 1993;20(suppl 3):S70-4.

35 Krupa DJ, Thompson JK, Thompson RF. Localization of a memory trace in the mammalian brain. Science 1993;260:989-91.

36 Small SL, Hlustik P, Noll DC, et al. Cerebellar hemispheric activation ipsilateral to the paretic hand correlates with functional recovery after stroke. Brain 2002;125:1544-57.

37 Brodal P. Principles of organization of the corticopontocerebellar projection to crus II in the cat with particular reference to the parietal cortical areas. Neuroscience 1983;10:621-38.

38 Roviller EM, Liang F, Babalian A, et al. Cerebellothalamocortical and pallidothalamocortical projections to the primary and supplementary motor cortical areas: a multiple tracing study in macaque monkeys. J Comp Neurol 1994;345:185-213.

39 Seitz RJ, Hoflich P, Binkofski F, et al. Role of the premotor cortex in recovery from middle cerebral artery infarction. Arch Neurol 1998;55:1081-8.
40 Reddy $\mathbf{H}$, Lassonde M, Bemasconi N, et al. An fMRI study of the lateralization of motor cortex activation in acallosal patients. Neuroreport 2000;11:2409-13.

41 Reddy H, Matthews PM, Lassonde M. Functional MRI cerebral activation and deactivation during finger movement. Neurology 2000;55:1244.

42 Weiller C, Ramsay SC, Wise RJ, et al. Individual patterns of functional reorganization in the human cerebral cortex after capsular infarction. Ann Neurol 1993;33:181-9.

43 Reddy H, Narayanan S, Arnoutelis R, et al. Evidence for adaptive functional changes in the cerebral cortex with axonal injury from multiple sclerosis. Brain 2000;123:2314-20.

44 Adcock JE, Wise RG, Oxbury JM, et al. Quantitative fMRI assessment of the differences in lateralization of language-related brain activation in patients with temporal lobe epilepsy. Neuroimage 2003;18:423-38.

45 Cohen MS, DuBois RM. Stability, repeatability, and the expression of signal magnitude in functional magnetic resonance imaging. J Magn Reson Imaging 1999; 10:33-40.

46 Pariente J, Loubinoux I, Carel C, et al. Fluoxetine modulates motor performance and cerebral activation of patients recovering from stroke. Ann Neurol 2001;50:718-29.

47 Riahi F, Zijdenbos A, Narayanan S, et al. Improved correlation between scores on the expanded disability status scale and cerebral lesion load in relapsing-remitting multiple sclerosis. Results of the application of new imaging methods. Brain 1998;121:1305-12.

\section{NEURONLINE}

\section{Baylor neurology case of the month: www.bem.tmc.edu/neurol/case.html}

nternet educational tools are often full of fancy graphics displays but short on thought. The Baylor College of Medicine's neurology department's case of the month site is the antithesis of this approach. Each month a case that residents have found interesting is posted on this simple, well thought out site.

First a detailed history and examination is presented for each case, from which it is sometimes possible to make a clinical diagnosis. It is usually necessary, however, to proceed to the test result page. Here, a plan of investigation can be drawn up, by choosing 14 tests from a list of about 80 possible laboratory, radiological, and neurophysiological studies. Radiological and pathological images can be reviewed and, at the cost of one investigation, an interpretation provided.

Hopefully, with a careful analysis of the history and examination findings, and interpretation of the test results, you can reach a diagnosis. Your diagnosis is then submitted by email. Later, the correct diagnosis is sent out and, if you sign up for the mailing list, you are told when new cases are posted. There is a short multiple choice test following each case. There are now more than 70 old cases to work through, each with a detailed discussion-anyone who enjoys clinical conundrums should start with case number 64 .

There are not many case based neurology websites, and of those that are available, this is by far the best. An old neurologist said

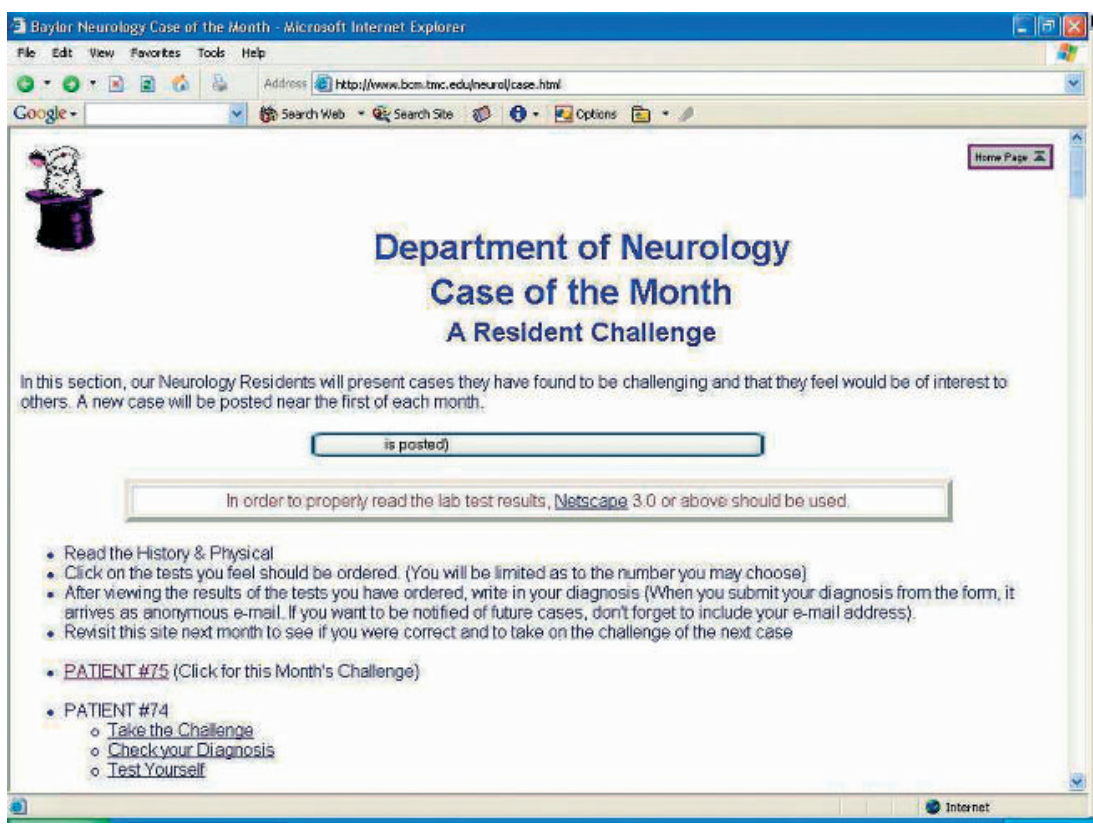

that given a spare half an hour, one should see a patient rather than read a paper-perhaps if there are no patients to hand 30 minutes at www.bcm.tmc.edu/neurol/case.html would be time well spent.

W Whiteley

Department of Clinical Neurosciences, Western General Hospital, Crewe Road, Edinburgh EH4 2XU, UK; ww@skull.dcn.ed.ac.uk 